\title{
Load Balancing Algorithm of Switched Dynamic Iteration
}

\author{
Chen Yansheng ${ }^{1,3, *}$, Wu Zhongkun ${ }^{1}$ and Ren Jiangtao ${ }^{2}$ \\ ${ }^{1}$ Guangdong Industry Technical College, Guangzhou, China 510300; ${ }^{2}$ School of Software, Sun Yat-Sen University, \\ Guangzhou, China 510275; ${ }^{3}$ Kun Shan University, Tainan, Taiwan 71003
}

\begin{abstract}
In order to reduce business latency and packet loss rate, to improve the throughput of integration of heterogeneous wireless network, to achieve load diversification and to improve end quality of service, while there are many problems on the dropout rate of the traditional load balancing algorithm in processing throughput, delay and business, therefore, gateway load balancing algorithm is proposed in this paper. All terminals in the access network can reflect the effectiveness of the average network load level of the network; this algorithm will gain weight of the load in the network of small business terminal to switch to the network load which is light. First, it defines the heterogeneous networks and network terminal payoff function utility functions which are used to characterize the experience and network QOS terminal load situation, and then presents the specific processes of the gateway load balancing algorithm. Finally, switching decisional load balancing algorithm, proposed by Yan X, etc. is compared with simulation experiments and it shows that: the proposed gateway load balancing algorithm has strong robustness to achieve network load balancing and to achieve a balanced use of network resource.
\end{abstract}

Keywords: Efficiency, loading differences, real-time business, switching decision.

\section{INTRODUCTION}

Load balancing is an important way, which makes full use of heterogeneous wireless network resources. Through the load balancing among the heterogeneous networks, the high probability of network load can be reduced, the overall utilization of network resources is improved and the blocking probability is reduced to provide users a better QOS, so the load balancing between the networks is also an important aspect of considering the access algorithm selected [1-3].

The user can only access a kind of network at the same time with the traditional hard load balancing service, which can not satisfy differentiation of the user service, resulting in partially utilized heterogeneous network resources and higher traffic blocking ratio. Literature [4] proposes the soft load balancing algorithm, which dynamically changes the best ratio of IP flow; when the network load is heavy, each network sub-flow rate of accessing users is consistently improve, while when the network load is light, there is a differences between the needs of users business rate and wireless resources [5-7].

The main purpose of heterogeneous networks is to achieve a variety of fast, reliable and secure exchange and transmission of information, that is, through the network to provide a wide range of high quality services, thus, business development, prosperity and evolution is the foundation and a key procedure of network development, prosperity and evolution [8-10].

This point is especially evident since 2000 , which is during a commercial $3 \mathrm{G}$ network and the development process of the Internet. As a whole, mobile communications operators can provide a variety of services through a variety of networks, and fully guarantee the user in the network to enjoy a variety of services, to improve the quality of the user experience. It is an inevitable tendency of development of wireless networks [11-14].

The integration of heterogeneous networks has become the developmental direction of next-generation wireless networks, wireless resources shared is the premier intention for the integration of heterogeneous wireless networks and reasonable heterogeneous network resource scheduling is a key technologies to achieve efficient radio resource sharing [1517]. Load balancing is determined by multiple servers in a symmetrical manner to form a collection of servers, each server has the equivalent status, and it can provide service outside independently without the aid of other servers. Through some sort of load balancing technology that is to be sent to external requests which are evenly distributed to the symmetrical structure of a server, and the server receives the request independently and responds to customers' requests [18]. Load balancing evenly gives out client requests to a server array, aiming at providing quick access to important data, to solve a large number of concurrent access to service issues. Network load balancing can effectively reduce the imbalance of resource utilization among heterogeneous networks, rational management of network resources is one important way. In the fusion of heterogeneous wireless network, terminals can carry out dynamic network choices, accessing and switching, to achieve load balancing of the RAN in heterogeneous radio access network (Radio access network, RAN), thereby improves network throughput and overall performance. Currently, the network load balancing research has focused on homogeneous network load balancing between different cells and certain types of load balanc- 
ing among heterogeneous networks. Depended on the implementation of existing research, methods can be divided into two categories: one is the accessing load balancing, the other is switched load balancing. Compared with the accesstype load balancing, switching load balancing has its quick response, fast convergence advantages. However, the key premise of load balancing switch is correct assessment of each cell load. For homogeneous network, the load of each cell is available and can be stated by unified indicators. However, in heterogeneous wireless network environment, the business type, resource allocation, and the assurance capabilities of service quality of the heterogeneous network, etc. are different. How to measure and compare the heterogeneous network load becomes a problem.

LOUHA K put forward the concept of soft load balancing - the downlink IP grouping into sub-streams, each substream accessing to different wireless network. This method can improve resource utilization in heterogeneous wireless networks. But when SON H and LOUHA K investigated the best diversion rates of in the case of specific network topology and ideal channel, the findings did not have universal applicability. Load balancing algorithm proposed by SHI Wenxiao, etc [19-22]. Presenting a method that can dynamically change optimal splitting ratio of IP flow; when the network load is heavy, it can constantly adjust the rate of user accessing to each network sub-flow. However, it did not consider that when the load is light, there is a difference between rate requirements of user business and wireless service. Meanwhile, Sun Zhuo also pointed out that as the radio resource units were appropriately allocated to the appropriate users to better meet user QOS, it will also improve the utilization of radio resources [23-25].

For the above what we has discussed, a gateway load balancing algorithm is put forward, which is based on the QOS (Quality of service, QOS), and the algorithm first defines a generalization for payoff function of network terminal and the utility function of heterogeneous networks respectively, to characterize the quality of service and network load terminal situation. Then, in a dynamic, iterative manner, the heaviest load of the network QOS gains and lower resource efficiency of terminal can be improved and scheduled to gain network lightest load in QOS revenue, to reduce load variation of between the networks and improve efficiency in the use of network resources.

This paper mainly made a work in the following areas expansively and innovatively:

(a) When dealing with throughput, latency and packet loss rate on business, the traditional load balancing algorithm in heterogeneous wireless network has many problems, so gateway load balancing algorithm is proposed. The QOS income of generalized users and network utility of the algorithm, which is based on the features of wireless business, are able to characterize the quality of service experience in the terminal network and network load conditions. And for a variety of heterogeneous network it is universal. Among them, the network utility's universality to heterogeneous network makes load conditions of each heterogeneous network comparable. TTERATIVE algorithm, by scheduling the QOS gains in the network with the heaviest load and the terminal with the lower resource efficiency to the network that is able to improve the QOS benefit with the lightest load, which can achieve switching load balancing among heterogeneous networks.

(b) In order to further validate the correctness and efficiency of the proposed gateway load balancing algorithm about the packet loss rate, packet delay and throughput performance, the author made a comparison with switching decisional algorithm proposed by Yan X for load balancing, and simulation experiments evaluated its performance of gateway load balancing algorithm based on QOS by using OMNET ++4.0 . Setting there are two types of 802.11 and 802.16 RAN in the system, each type of RAN has 2. Both algorithms at packet loss rate of a constant rate traffic remained unchanged, but the proposed algorithm in this paper significantly reduces packet loss rate of the real-time variable rate business and packet delay; as the load increases, the proposed algorithm can significantly improve the whole network throughput. Experiments show that: QOS-based gateway load balancing algorithm has strong robustness, and it can significantly improve the integration of heterogeneous network throughput, reduce business latency and packet loss rate. It can reach the effects of improving efficiency of network resource utilization.

\section{PROPOSED SCHEME}

The core idea of algorithm is: the QOS income of terminal end can reflects the current level of network quality of service received, the greater the benefits is, the better the resulting quality of service is, and vice versa; the average QOS benefit of all terminals in a accessing network (hereinafter defined as a network utility) can reflects the load level of the network, the larger the average gain of the terminal is, the load on the network is lighter, and vice versa. In order to achieve diversification and to increase the service quality of load terminal, the algorithm will switch small businesses with gain heavy load in the network to a lighter terminal network load.

In order to carry out the terminal to select the RAN and its access effectively and dynamically, the multimode terminal will experience unified management from the network side, which will be completed by the network side management entity (Network side manager, NSM). The terminal will interact with NSM through the terminal-side management entity (Terminal-side manager, TSM), dynamically achieving refactoring of switching/accessing. The interaction between NSM and TSM is completed through management control channel (Management and control channel, MCC). NSM is deployed in the core network, and is shared by a plurality of RAN. RAN will convey each context information to NSM, then NSM transfer each context information of RAN to the terminal for the decisions through the MCC downlink transmission. TSM of each terminal sends context information of the terminal through uplink transmission of the MCC to NSM. Based on context information of RAN and terminal, NSM uses the appropriate network selection algorithm to develop strategies and policies issued under the various terminals. Terminal then chooses according to their needs and network reconfiguration decisions and configures 
Table 1. Wireless network traffic types and their QOS requirements.

\begin{tabular}{|c|c|c|c|c|c|}
\hline \multirow{2}{*}{ Real-time business } & Rate of Change & The Minimum Rate & Maximum Rate & $\begin{array}{c}\text { Time Delay } \\
\text { Requirements }\end{array}$ & A Typical Business \\
\cline { 2 - 5 } & constant & $r_{\text {min }}=r_{\text {max }}$ & $r_{\text {min }}=r_{\text {max }}$ & $d_{\text {max }}$ & VoIP \\
\hline \hline The real-time business & variable & $r_{\text {min }}$ & $r_{\text {max }}$ & MPEG \\
\hline
\end{tabular}

to access the appropriate RAN. The paper will assume the terminal in the network / inter-cell handover fast enough, and thus, the load balancing process, due to switching delay caused by the upper risk of business disruption can be ignored.

\subsection{QoS Revenue and RAN Utility}

Different types of wireless network services have different QOS requirements. Based on the characteristics of the various services, wireless services can be classified into three types of basic services, as shown in Table 1.

According to real-time requirements, the wireless business can be classified into real-time business (Real time, RT) and non-real-time service (Non-real time, NRT). According to whether the rate can change or not, real-time service has been divided into two types of constant rate and variable rate. Minimum service time at a constant rate $\min$ and the maximum rate requirements $\max$ are equal, that is the rate are unchanged. The variable rate real-time service is possessed of the minimum and maximum rate requirements. When the waiting time of a real-time service exceeds the maximum packet delay tolerance $d_{\max }$, the packet will be discarded. Non-real-time service needs not to delay, and the minimum bandwidth can become zero.

In order to characterize the obtained terminal from the current network quality of service, based on the QOS requirements of different business, $s i_{\text {gmond }}$ function to construct the terminal payoff function is used. So suppose $\mathrm{J}$ is the set of kinds of RAN, $I$ represents the set of terminals in the network, defining an accessing terminal $i \in I$ of RAN $j \in J$ QOS benefit function is as follows:

$U_{i j}=\left\{\begin{array}{l}\left(1-\frac{1}{1+\exp \left(-\partial_{m} \times \frac{d_{i j}-d_{i j}^{e}}{d_{i j}^{\max }-d_{i j}^{m}}\right)}\right) \cdot B T \\ \left(1-\frac{1}{1+\exp \left(-\partial_{m} \times \frac{d_{i j}-d_{i j}^{e}}{d_{i j}^{\min }-d_{i j}^{r}}\right)}\right) \cdot B R T\end{array}\right.$

For real-time services, at the premise to meet the minimum bandwidth, using an average delay $d_{i j}$ to measure user gains, the smaller the time delay is, the higher the gain is; for non-real-time services to the user, using average speed $r$ of the user gains to measure, the larger the rate is, the higher the income is;

$$
\frac{d_{i j}-d_{i j}^{e}}{d_{i j}^{\max }-d_{i j}^{r}}
$$

If delay for real-time services the normalized, wherein $d_{i j}^{e}$ denotes the expectancy of average delay of real-time services:

$$
\frac{\beta_{i j}-\beta_{i j}^{e}}{\beta_{i j}^{\max }-\beta_{i j}^{r}}
$$

As the normalization about Non-real-time services' rate, it helps to ensure a minimum rate of non-real-time services; $\beta_{i j}$ and $\beta_{i j}$ is constant parameters, which determines the steepness of the curve of the function, the larger the value is, the steeper the curve changes, the higher the sensitivity to the end quality of service is. Formula (1) as defined in revenue function reflects QOS-awareness of terminal, the function maps a plurality of QOS parameters with reasonable perception or experience for the user to QOS level, gives a measurement of the QOS of different users by using uniform quantization levels standards.

To characterize the load level of the accessing network, the wireless access network defines the utility of all the terminals connected to the network average of QOS benefit. Suppose at a time, a terminal can only access a RAN, then the gain RAN $J \in I$ can be expressed as:

$$
R_{i j}=\left\{\begin{array}{c}
\frac{\sum_{j \in i} \alpha_{i j} n_{i j}}{\sum_{J \in I} \alpha_{i j}}, \sum_{I \in J} \partial_{i j} \neq 0 \\
1, \quad \sum_{i \in j} \partial_{i j}=0
\end{array}\right.
$$

wherein, $\partial_{i j}=\left\{\begin{array}{c}1 \text { terminal } i \text { int } o R A N_{j} \\ 0 \text { other }\end{array}\right.$

Obviously, the heavier network load will result in lower average QOS benefit of terminal, otherwise the terminal average QOS gains will be higher. Therefore, the average QOS benefit of the terminal, namely the network load of the network utility can reflects the situation. Network utility is higher, indicating that the network load is lighter, otherwise it indicates the network load is heavier.

\subsection{Load Balancing Algorithm Design}

When the integration of heterogeneous networks presents a state of uneven load distribution, load balancing algorithm 
based on QOS -awareness will be triggered. Network load distribution algorithm will undergoes iterative adjustments. Each iteration process can be described as:

(1) Select a heavy-duty accessing network from an entire network called upon burdens;

(2) Select offloading terminal from the accessing network, as a switch to a different accessing network of the terminal, called subject to switch the terminal;

(3) Select an accessing network with a lighter load from subject to switch the terminal, switched to as the purpose of switching the terminal accessing network, called upon subject to increasing negative network;

(4) If the selected network can be added to improve the negative terminal, the quality of service to be switched, then the switch terminal is switched to be negative to the network which is to be added into the next iteration; otherwise, the terminal remains in the burden upon the network, then offloading network is selected to be another terminal as a terminal to be switched and it is performed (3) and (4) again. If the burdens upon all terminals in the network choose to stay in the accessing network to be reduce the burden, it is considered as a more balanced distribution of network load that can no longer improve the algorithm ends.

The core of load balancing algorithm based on QOS awareness is: Web-based utility accessing network selection to be burdens, to be based on the weighted QOS gains selection of subjecting to switch terminals, terminal and networkbased utility and accessibility testing negative access network to be increased selection.

\subsubsection{Web-Based Utility Accessing Network Selection to be Burdens}

In order to make load balancing rapidly converge, algorithms in each iteration find the heaviest load of the whole network accessing network, and select one of the appropriate terminal switch to other accessing networks.

According to the definition and analysis of the effectiveness of the network utility in the section of 3.1, it can effectively reflect the access network load conditions. Therefore, the algorithm selects the lowest utility network accessing network as a network to be burdens. If pending burdens network denoted $J \in I$, then:

$$
\begin{aligned}
& r^{n}=\arg \min _{j \in J}\left\{\begin{array}{c}
- \\
m_{j}
\end{array}\right\} \\
& r^{i}=\arg \min \left\{\begin{array}{c}
- \\
m_{i}
\end{array}\right\}
\end{aligned}
$$

\subsubsection{The Weighted Selection of Subjecting to Switch Ter- minal about QoS Benefit to be Based on}

In order to make load balancing rapidly converge, algorithms in each iteration find the heaviest load of the whole network accessing network, and select one of the appropriate terminal switch to other accessing networks. When the accessing network subject to reducing the burdens selects to switch's terminal, it not only needs to consider the quality of service experience of terminal at the corresponding to the accessing network, namely it should choose a lower the QOS experience in order to the user expects to switch to other network medium to get improved; and it needs to consider the users' radio resource utilization efficiency to be burdens in the accessing network, which should tend to choose the channel conditions of poor terminal to switch to other networks, in order to terminate their treatment on resources inefficient usage in subjecting to reducing the burdens in the accessing network channel. Considering two aspects as defined in the terminal $i$ in the weighted QOS benefit function of the RAN $\mathrm{j}$ is as follows:

$$
\begin{aligned}
& J_{i j}^{*}=a_{i j} \times J_{i j} \\
& J_{i j}^{*}=\left(a_{i j}+J_{i j}\right)
\end{aligned}
$$

Wherein the weighting factor $a_{i j}$ reflects resource utilization efficiency of the terminal $i$ in the radio accessing network $j$, which is defined as:

$$
\begin{aligned}
& a_{i j}=\frac{J_{i j}\left(e_{i j}\right)}{J_{i j}^{\max }} \\
& e_{j}^{i}=\sum_{g}^{a_{i j}} \beta u_{i}^{j}
\end{aligned}
$$

$J_{i j}\left(e_{i j}\right)$ is the actual rate that each unit bandwidth obtained for the accessing terminal $i$ to the accessing network $\mathrm{j}$ ; it indicates channel condition between the terminal $i$ and accessing network $\mathrm{j} ; R_{i, j}^{\max }$ presents the maximum rate can obtain per unit of bandwidth from accessing terminal $i$ to the accessing network $\mathrm{j}$ theoretically. Thus, the greater $a_{i j}$ is, indicating that the channel conditions is better between terminals $i$ and accessing network $\mathrm{j}$ the terminal $i$ has a good utilization efficiency in the accessing network channel.

Considering the quality of service experience and radio resource utilization of terminal accessing network, algorithm selects the lowest weighted terminal of subjecting to reducing the burdens based on QOS gains as a terminal to be switched. So suppose $e_{j}^{i}$ is the set of $e_{j}^{i}$, all of the access terminals, then $e^{i}$, the terminal to be switched, selected in the offloading accessing network, is supposed to be met:

$$
\begin{gathered}
e^{i}=\underset{i \in j^{i}}{\arg \min }\left\{e_{i j}^{*} n\right\} \\
. t_{j}^{i}\left(e_{i j}^{*} n\right)
\end{gathered}
$$

\subsubsection{The Selection of the Accessing Network Subject to be Negative Growth}

When the terminal to be switched $e_{j}^{i}$ selects $e^{i}$, the intending accessing network, for the purpose of switching accessing networks, on the one hand, it needs to identify whether the terminal $e_{j}^{i}$ is within the coverage area of the accessing network $e^{i}$, and the remaining capacity of $e^{i}$ can 
meet the minimum rate requirements of $e_{j}^{i}$. That the intending accessing network $e^{i}$ shall meet:

$\left\{\begin{array}{c}e_{i^{n}}\left(r_{n}^{i}, e^{i}\right) \succ 0 \\ e_{i j}^{\max }+\sum_{j \in i} \partial_{i j}^{\min } \leq r_{i j}^{\max }\end{array}\right.$

Among which, $r_{i j}^{\max }$ represents the maximum network capacity of accessing networks $e^{i}$.

On the other hand, for the benefit of rapid convergence load balancing, you should choose a lighter network load to be switched as the intending accessing network. Thus, the algorithm for all the condition (7) of the accessing network ( except the accessing terminal subject to being switched and reducing the burdens) of a collection (referred to as $e_{j}^{i}$ ), select the accessing network with highest utility network, as the purpose of the accessing network terminal to be switched, the network to be negative growth can be expressed as:

$e_{j}^{i}=\underset{j \in i^{\min }}{\arg \max }\left\{\bar{m}_{j}\right\}$

\subsubsection{QoS-awareness Load Balancing Algorithm}

Based on the above analysis, the designation of gateway load balancing algorithm process is as follows:

Initialization: Let $\mathrm{J}$ represents algorithm converged network within the scope of all the heterogeneous collection of RAN, I represents the set of all terminals in the network; / / Algorithm carries through load balancing to a certain geographical area of heterogeneous converged network.

Step 1: Select (3) to satisfy the access network $e^{i}$ as a network to be the network subject to reducing the burdens; $e_{j}^{i}$ as a collection of all terminals $e^{i}$ connected, optionally switch the terminal to be set $e_{j}^{i}={ }^{-i} e_{j}$.

/ / selects the heaviest accessing network, ready to be moved to another part of the load in the accessing network.

Step 2: In $e_{j} e_{j}$, select the terminal $e^{i}=\underset{i \in m}{\arg \min }\left\{e_{i j}^{*}\right\}$ as to be switching terminals.

/ / selects the terminal with a lower service quality of experience and resource utilization, ready to be switched to another accessing network.

Step 3: Among other accessing network options except $e^{i}$, select $e_{j}^{i}$ to meet (7) formula and (8) as accessing network to be negative growth.

/ / selects an accessing network terminal with a lighter load to be switched as the intending accessing network.

Step 4: Switch the terminal to be switched $e^{i}$ to the accessing network to be negative growth $e_{j}^{i}$, if $e_{j}^{i} \succ e_{i^{h}, j^{n}}$

The terminal $e^{i}$ switch to $e_{j}^{i}$, then return to step 1 ; otherwise terminal $e^{i}$ going back to $e_{j}^{i}$, while the optional terminal to be switch can is updated to $e_{j} e^{i}=e_{j}^{i}-\left\{e^{i}\right\}$ and perform step 5 .

/ / If the accessing network can be increased to improve the negative terminal of the quality of service to be switched, the adjustment is successful and re-select the accessing network of subjecting to reducing the burdens to carry out a new round of adjustment; otherwise the terminal to be switched from the optional subject can be removed from the terminal concentrator.

Step 5: If $e_{j} e_{j} \neq 0$, then go back to step 2;

/ / From the accessing network of pending burdens to be switched of the terminal concentrator, choose optional weighted QOS benefit low end times, try to switch to another accessing network. Otherwise, the algorithm terminates.

/ / Accessing network to reduce the burden of all the terminals are choose to stay in the accessing network, unable to further improve the extent of the network load balancing, it can be considered that the state has achieved load balancing.

It can be seen that network utility and terminal benefits can achieve quantitative balance from the algorithm through a unified QOS revenue function designation. By choosing and switching network and terminal QOS benefit between the network utility and terminals tends to equilibrium. The cost is based on algorithm designation, need to run on the network and terminal management entity execution algorithm, and the corresponding QOS information and interactive network selection strategy. Network side and terminal side executing algorithm, network management entity, can be naturally and easily completed by heterogeneous network convergence in the framework of NSM and TSM, interaction of information and strategies can be completed by the contextual messages of the MCC. In the current level of technology, NSM and TSM itself has a powerful computing capabilities, and load balancing does not require strict real-time and interactive information and data of selection strategies for QOS is a small amount, therefore the proposed algorithm can obtain improved network and terminal performance significantly at little cost.

\section{EXPERIMENTAL RESULTS}

By using OMNET ++4.0 , it can evaluate its performance of load balancing algorithm based on QOS-awareness. Supposing that, there are two types of 802.11 and 802.20 RAN in the system, each type of RAN has 2. Simulation only consider the upstream traffic, 802.20 RAN adopts the TDD mode of single carrier, each TDD frame in the uplink and downlink frames has each half; 802.23 RAN uses DCF mode. There are 30 supposed user (terminal), each user uses only one business, each has 10 business users. Setting the density of user in 802.20 RAN, the user from the SNR to the 
Table 2. Experimental parameters.

\begin{tabular}{|c|c|c|c|}
\hline Parameter Names & The Parameter Value & Parameter Names & The Parameter Value \\
\hline \hline The 802.20 frame length/ms & 1 & Packet length/bit & 1000 \\
\hline The 802.20 disabled when the time/ms & 0.0002 & $w_{x y} / \mathrm{ms}$ & 0 \\
\hline 802.20 Frame when the number of disabled & 5000 & $w_{x y} / \mathrm{ms}$ & 0.53 \\
\hline The 802.23 disabled when the time/ms & 0.023 & $\eta_{x}$ & 0.02 \\
\hline The SIFS length/ms & 0.010 & $\eta_{y}$ & \\
\hline The DIFS length/ms & 0.053 & & \\
\hline
\end{tabular}

Table 3. Simulation results.

\begin{tabular}{|c|c|c|c|c|c|c|c|c|c|}
\hline \multicolumn{2}{|l|}{ SN } & 1 & 2 & 3 & 4 & 5 & 6 & 7 & 8 \\
\hline $\begin{array}{l}\text { Real time constant variable bit rate } \\
\text { of packet loss rate }(\%)\end{array}$ & QALBA & 0 & 0 & 0 & 0 & 0 & 0 & 5.4 & 5.5 \\
\hline $\begin{array}{l}\text { The average packet delay of time } \\
\text { business (ms) }\end{array}$ & HDLBA & 0 & 0 & 0 & 0 & 73 & 82 & 93 & 102 \\
\hline \multirow{2}{*}{$\begin{array}{l}\text { The throughput of whole network } \\
\text { (Mbit/s) }\end{array}$} & HDLBA & 3.6 & 6.2 & 8 & 8.6 & 7.3 & 7.1 & 9.2 & 9.6 \\
\hline & QALBA & 3.7 & 6.6 & 8.2 & 10 & 11 & 13 & 16 & 16 \\
\hline
\end{tabular}

RAN randomly changed. Users can access a RAN at the same moment. Service packets arrive to Poisson distribution. The minimum rate of Variable rate services reached $1 / 2$ of average rate. Users initially access network, whose selections are based on SINR (Signal-to-interference plus noise ratio, SINR) criterion, that is, channel conditions between the user and the various RAN, dynamically select the highest RAN of SINR to access, while according to network selection principle of $802.20 / 802.23$ converged network users to access, business users access to 802.20 networks real-timely at a constant rate. The associated parameter settings used by network simulation are shown in Table 2.

In order to verify the performance of the proposed gateway load balancing algorithm, by using handover decision load balancing algorithm (Handoff decision load-balancing algorithm, HDLBA) proposed by Yan X, etc as a compared algorithm, the packet loss rate, packet delay and throughput performance were compared.

Table 3. Shows the simulation results of the comparison.

Table 3 parts for Real time constant variable bit rate of packet loss and The average packet delay of time business show that the changing situation of real time constant bit rate and real time constant variable bit rate, average packet loss rate and packet delay variation when the average rate of each connection from $100 \mathrm{Kbit} / \mathrm{s}$ increased to $450 \mathrm{Kbit} / \mathrm{s}$. As can be seen, the proposed gateway load balancing algorithm significantly reduces the real-time variable rate, packet loss rate and packet delay; while the average delay and packet loss rate of real-time business a constant rate in the two algo- rithms is basically to keep unchanged. It is because the priorities safeguard mechanism of 802.16 networks for realtime business with a constant rate can guarantee QOS service of real-time at a constant rate, while the two algorithms, which can ensure a constant rate of real business users, always stay in the 802.16 network, and get a guaranteed quality of service.

Table 3 part of the throughput of whole network, shows as the load increases, the proposed gateway load balancing algorithm can significantly improve the throughput of the whole network.

Simulation results show that: QALBA algorithm can balance the network load, and compared to the traditional MLB algorithm and DLBD algorithm, the average blocking rate of packet service and the throughput performance have been improved significantly, and the robustness of algorithm is really strong, which can achieve network load balancing to achieve a balanced use of network resources.

\section{CONCLUSION}

For the integration of heterogeneous wireless network communication scenario, the network load balancing algorithm was proposed based on the QOS, which is fallen into switchable load balancing algorithm. Algorithm is based on QOS gains and network utility of a generalized feature for wireless business users, and it is able to characterize the quality of service terminal network experience and network load conditions, and it is universally applicable for a variety 
of heterogeneous network. Among them, the universality of network utility to heterogeneous network makes heterogeneous network load comparability comparable, able to achieve switching between heterogeneous network load balancing. Simulation results show that the proposed gateway load balancing algorithm can improve integration of heterogeneous network throughput, reduce business latency and packet loss rate, with strong robustness to achieve network load balancing and to achieve a balanced use of network resources.

\section{CONFLICT OF INTEREST}

The authors confirm that this article content has no conflict of interest.

\section{ACKNOWLEDGEMENTS}

This work was financially supported by Guangdong Industry Technical College Foundation (KJ2013110).

\section{REFERENCES}

[1] K. Suhairi, and F.L. Gaol, "The measurement of optimization performance of managed service division with ITIL framework using statistical process control," Journal of Networks, vol. 8, no. 3, pp. 518-529, 2013.

[2] Y. Guang, Y. Zhu, C. Gu, J. Fei, and X. He, "A framework for automated security proof and its application to OAEP," Journal of Networks, vol. 8, no. 3, pp. 552-558, 2013.

[3] M.J. Mirza, and N. Anjum, "Association of moving objects across visual sensor networks," Journal of Multimedia, vol. 7, no. 1, pp. 28, 2012.

[4] M. J. Mirza, and N. Anjum, "Association of moving objects across visual sensor networks," Journal of Multimedia, vol. 7, no. 1, pp. 28, 2012.

[5] Z. Duan, Z. Yuan, X. Liao, W. Si, and J. Zhao, "3D tracking and positioning of surgical instruments in virtual surgery simulation," Journal of Multimedia, vol. 6, no. 6, 502-509, 2011.

[6] S.H. Tang, M.C. Chen, Y.S. Sun, and T. Zsehong, "A spectral efficient and fair user-centric spectrum allocation approach for downlink transmissions," IEEE., Globecom., pp. 1-6, 2011.

[7] C. Xu, J.H. Lu, and Y. Zhen, "An Experiment and analysis for a boiler combustion optimization on efficiency and NOx emissions," Boiler Technology, vol. 37, no. 5, pp. 69-74, Oct. 2006.

[8] L.G. Liang, Y. Meng, and S.L. Wu. "Operation optimization for retrofitted $1025 \mathrm{t} / \mathrm{h}$ boiler and experimental study on its NOx emission," Thermal Power Generation, vol. 42, no. 1, pp. 63-66, Jan. 2013.

[9] Z.Y. Gao, Z. Guo, J.Q. Hu, X. Wu, and X. Wang, "Multi-objective combustion optimization and flame reconstruction for W shaped boiler based on support vector regression and numerical simulation". Proceedings of the CSEE, vol. 31, no. 5, pp. 13-19, May 2011.
[10] K. Ruttik, K. Koufos, and R. Janttir, "Model for computing aggregate interference from secondary cellular network in presence of correlated shadow fading," In: IEEE, $22^{\text {nd }}$ International Symposium on Personal, Indoor and Mobile Radio Communications, 2011, pp. 433-437.

[11] D.L. Sun, X.N. Zhu, Z.M. Zeng, and S.H. Wan, "Downlink power control in cognitive femtocell networks," In: IEEE International conference on wireless communications and signal processing, 2011, pp. 1-5.

[12] N. Omidvar, B.H. Khalaj. "A game theoretic approach for power allocation in the downlink of cognitive radio networks," In: IEEE $16^{\text {th }}$ CAMAD, 2011, pp. 158-162.

[13] D. Xu, Z.Y. Feng, Y.Z. Li, P. Zhang, "Fair Channel allocation and power control for uplink and downlink cognitive radio networks," In: IEEE Workshop on mobile computing and emerging communication networks, 2011, pp. 591-596.

[14] W.Q. Yao, Y. Wang, and T. Wang, "Joint optimization for downlink resource allocation in cognitive radio cellular networks," In: IEEE $8^{\text {th }}$ Annual IEEE consumer communications and networking conference, 2011, pp. 664-668.

[15] J. Naereddine, J. Riihijarvi, and P. Mahonen. "Transmit power control for secondary use in environments with correlated shadowing," In: IEEE, ICC2011 Proceedings, 2011, pp. 1-6.

[16] R. Berangi, S. Saleem, M. Faulkner, and W, Ahmed, "TDD cognitive radio femtocell network (CRFN) operation in FDD downlink spectrum," In: IEEE, $22^{\text {nd }}$ International Symposium on Personal, Indoor and Mobile Radio Communications, 2011, pp. 482-486.

[17] W. Ahmed, J. Gao, S. Saleem, and M. Faulkner, "An access technique for secondary network in downlink channels," In: IEEE, $22^{\text {nd }}$ International Symposium on Personal, Indoor and Mobile Radio Communications, 2011, pp. 423-427.

[18] G. Isabel, and S. Ismael, "Variable selection for multivariate statistical process control". Journal of Quality Technology, Milwaukee: vol. 42 , no. 3, p. $242,2010$.

[19] R.T.S Ramakrishnan, and A.V. Thakor, "Information reliability and a theory of financial intermediation", The Review of Economic Studies, vol. 51, no. 3, pp. 415-432, 1984.

[20] S. Rick, "Preventive maintenance your SPC," Journal of Quality Technology, Automated Precision Inc., vol. 49, no. 12, p. 13, 2010.

[21] R. William, and J.R. Kinney, "Information Quality Assurance and Internal Control", McGraw-Hill: USA, 2000.

[22] M. Backes, M. Berg, and D. Unruh, "A formal language for cryptographic pseudocode," In: $15^{\text {th }}$ International Conference on Logic for Programming, Artificial Intelligence and Reasoning, LPAR 2008, volume 5330 of Lecture Notes in Computer Science. Springer: US, 2008, pp. 353-376.

[23] D. Nowak and Y. Zhang, "A calculus for game-based security proofs," In: Provable Security - $4^{\text {th }}$ International Conference, ProvSec 2010, Malacca, Malaysia, October 13-15, 2010.

[24] G. Barthe, B.Gregoire, S.Zanella, and Beguelin, "Formal certification of code-based cryptographic proofs," In: Proceedings of the $36^{\text {th }}$ ACM Symposium on Principles of Programming Languages, ACM Press, 2009, pp. 90-101.

[25] G Barthe, B. Gregoire, S. Heraud, S. Zanella, and Beguelin, "Computer-Aided Security Proofs for the Working Cryptographer," In: Advances in Cryptology - CRYPTO, vol. 6841 of Lecture Notes in Computer Science, Springer, 2011, pp. 71-90.

\footnotetext{
Received: September 16, 2014

Revised: December 23, 2014

Accepted: December 31, 2014

(C) Yansheng et al.; Licensee Bentham Open.
}

This is an open access article licensed under the terms of the Creative Commons Attribution Non-Commercial License (http://creativecommons.org/licenses/by-nc/3.0/) which permits unrestricted, non-commercial use, distribution and reproduction in any medium, provided the work is properly cited. 